\title{
QUALIDADE DE VIDA NO TRABALHO DO AGENTE COMUNITÁRIO DE SAÚdE
}

\author{
QUALITY OF LIFE IN THE WORK OF COMMUNITY HEALTH WORKERS
}

\author{
Ricardo Goes de Aguiar \\ Programa de Pós-Graduação em \\ Epidemiologia \\ Faculdade de Saúde Pública \\ Universidade de São Paulo \\ Curso de Fisioterapia \\ Universidade Federal de Sergipe \\ Rayane Rabelo Ferraz Viana \\ Neidimila Aparecida Silveira \\ Giselle de Carvalho Brito \\ Adriana Andrade Carvalho \\ Curso de Fisioterapia \\ Universidade Federal de Sergipe
}

\author{
Contato \\ Ricardo Goes de Aguiar \\ Rua Y, 959 - Summerville - casa 69 \\ Aruanda \\ Aracaju - SE \\ CEP: $49000-480$ \\ E-mail: ricardoaguiar@ufs.br
}

\section{RESUMO}

A singularidade do trabalho do Agente Comunitário de Saúde (ACS) como mediador do acesso a direitos sociais na região em que vive pode levar à sobrecarga física e mental. O objetivo deste trabalho foi analisar a Qualidade de Vida no Trabalho dos ACS que atuavam no município de Lagarto, Sergipe. Trata-se de um estudo transversal com ACS com pelo menos um ano de atuação na função no município, abordando Qualidade de Vida no Trabalho pelo modelo de Walton e aspectos socioeconômicos. Os dados foram digitados, e a análise estatística descrita foi realizada utilizando o EPi Info, versão $6.0 \mathrm{~d}$. A maioria dos participantes era do sexo feminino $(77,5 \%)$, com ensino médio completo $(68,3 \%)$, residindo na área de abrangência da unidade (82,5\%). Estavam insatisfeitos ou muito insatisfeitos com o salário, benefícios extras, distribuição dos Equipamentos de Proteção Individual, oportunidade de crescimento profissional, treinamentos e respeito aos direitos do trabalhador. Por outro lado, estavam satisfeitos ou muito satisfeitos com a importância do trabalho, relacionamento com colegas e chefes, comprometimento com o trabalho, respeito à individualidade e relevância social. Espera-se que o estudo subsidie ações destinadas à melhoria das condições de vida e trabalho desses trabalhadores.

Palavras-chave: Saúde da Família. Agentes Comunitários de Saúde. Qualidade de Vida. Satisfação no Emprego. 
The uniqueness of Community Health Workers' (CHW) work as a mediator to access social rights in their localities may lead to physical and mental overload. The aim of this study was to analyze the quality of life of the CHW in their work in the city of Lagarto, Sergipe, Brazil. A cross-sectional study was performed with CHA that worked at least one year in the city, by addressing the quality of life at work with the Walton's model and socioeconomic aspects. The data were entered, and the described statistical analysis was performed using EPi Info (version 6.0 d). Most participants were female $(77.5 \%)$, with a high school degree $(68.3 \%)$, and living near the primary healthcare unit area $(82.5 \%)$. The participants were dissatisfied or very dissatisfied with the salary, extra benefits, distribution of individual protection equipment, professional growth opportunities, training, and respect for workers' social rights. Nevertheless, they were satisfied or very satisfied with the importance of their work, relationships with colleagues and bosses, commitment to work, respect for individuality, and social relevance. Thus, the present study is expected to subsidize actions aimed at improving the living and working conditions of those workers.

Keywords: Family Health. Community Health Workers. Quality of Life. Job Satisfaction.

\section{INTRODUÇÃO}

A estruturação do Sistema Único de Saúde (SUS) se traduziu em transformações no sistema brasileiro de proteção social em saúde. Nesse cenário, a saúde da família surgiu como estratégia de reorientação do modelo de atenção do SUS, a partir da Atenção Primária à Saúde (APS), baseada em equipes mínimas compostas por médico, enfermeiro, auxiliar de enfermagem e Agentes Comunitários de Saúde (ACS). A proposta inicial do Programa de Agentes Comunitários de Saúde (PACS) era estender a cobertura dos serviços de saúde e, consequentemente, reduzir a mortalidade materna e infantil, principalmente nas áreas mais carentes das regiões Norte e Nordeste ${ }^{1-6}$.

O ACS foi reconhecido como profissional de saúde, sendo-lhe conferidas atividades de promoção da saúde e prevenção de doenças, por meio de ações individuais ou coletivas, de acordo com os princípios e diretrizes do SUS. Esse profissional representa um elo que possibilita a confiança e o vínculo entre a equipe de saúde e a comunidade assistida. Tem potencialidade para agregar saberes acerca do processo saúde-doença, assumindo a condição de tradutor do conhecimento científico em popular e colaborando para o acesso da comunidade aos serviços de saúde 7-10. $^{7}$.

Entre as suas atribuições, estão o mapeamento da área; cadastramento das famílias e atualização permanente; identificação de áreas de risco; realização de acompanhamento mensal das famílias sob sua responsabilidade; desenvolvimento de ações de educação e vigilância à saúde e repasse da dinâmica social da comunidade para os outros membros da Equipe de Saúde da Família (ESF) ${ }^{11,12}$.

Deve ser considerada a singularidade centrada no trabalho do ACS, que, por sua vez, exige atenção a inúmeros aspectos das condições de vida da população. Sua identidade comunitária e seu papel social diferenciam-no dos demais profissionais de saúde, principalmente por atuar como mediador e facilitador do acesso a direitos sociais. É um profissional que provém das classes populares e que possui características próprias, tendo em vista que atua no local que reside ${ }^{13,14}$.

Esse personagem tem uma missão social representada por sua identificação com a comunidade, seus valores, costumes, linguagem e o poder de liderança atribuído, com potencial possibilidade de promover melhoria nas condições 
de vida e saúde da população, o que torna tênue a linha que separa o profissional do morador e do usuário do serviço no qual trabalha ${ }^{15}$.

O trabalho desse profissional pode ser comprometido por fatores como as responsabilidades, condição salarial, resistência da população, além da inexistência de limites entre o ambiente de trabalho, seu conteúdo e o seu local de moradia, implicando sobrecarga tanto física quanto mental ${ }^{16}$.

Diante disso, a Qualidade de Vida no Trabalho (QVT) é considerada fator relevante na análise das condições de trabalho, relacionando variáveis ambientais, comportamentais e organizacionais, a fim de possibilitar humanização nos setores de trabalho, o que, consequentemente, implica a obtenção de resultados satisfatórios tanto para o trabalhador como para a instituição ${ }^{17,18}$.

Alguns modelos estruturais abordam a QVT utilizando indicadores de satisfação ou insatisfação, como o proposto por Walton, segundo o qual o conceito de QVT está calcado na humanização do trabalho e responsabilidade social da empresa, envolvendo necessidades e anseios do indivíduo, por meio de um processo de reestruturação e organização do trabalho, associado à formação de equipes com maior poder de autonomia. Para ele, deve existir um equilíbrio entre trabalho e outras esferas da vida ${ }^{19,20}$.

Para a avaliação da QVT, Walton elenca oito categorias: compensação justa e adequada, condições de trabalho, uso ou desenvolvimento das capacidades, oportunidade de crescimento e segurança, integração social na organização, constitucionalismo, trabalho e vida e relevância social ${ }^{21}$.

Nesse sentido, o estudo teve como objetivo analisar a QVT dos ACS do município de Lagarto, Sergipe (SE).

\section{MÉTODOS}

Realizou-se um estudo de abordagem quantitativa, transversal e descritivo. A população do estudo correspondeu a 135ACS, que atuavam há pelo menos um ano na função no município de LagartoSE e que aceitassem participar voluntariamente da pesquisa. Esses ACS estavam distribuídos em 18 equipes entre PACS e Estratégia Saúde da Família. Participaram da pesquisa 120 ACS, representando uma perda de $11,1 \%$.

A coleta de dados foi realizada por meio de questionários aplicados por entrevistadores treinados na própria unidade em que o profissional atuava, em local que garantisse a privacidade do participante, no período de agosto a outubro de 2014 . Foram utilizados dois questionários: socioeconômico e de Avaliação da Qualidade de Vida no Trabalho de Walton.

O questionário socioeconômico desenvolvido pelo grupo de pesquisadores do Programa de Educação pelo Trabalho para a Saúde (PET-Saúde) da Universidade Federal de Sergipe - campus Lagarto teve como objetivo caracterizar o perfil do ACS. Foram abordadas as condições de saúde e de trabalho, constando as variáveis: sexo, idade, estado civil, condições de moradia, escolaridade, raça, renda familiar, participação econômica, condições de deslocamento para o trabalho, prática de atividade física, quantidade de famílias que acompanha, visitas domiciliares, participação em capacitações e treinamentos.

O segundo instrumento de coleta de dados, o modelo Walton de Qualidade de Vida no Trabalho, foi escolhido pela sua abrangência e aplicabilidade no contexto. É um modelo multidimensional e autoexplicável, que utiliza como referência as 
percepções das últimas duas semanas.

O questionário é composto por oito domínios, e suas respostas abordam as variáveis da escala de resposta psicométrica de Likert, alternando desde muito insatisfeito a muito satisfeito, com cinco níveis cada uma, e domínios medidos em direção positiva. Assim, escores mais altos indicam melhor avaliação.

Os dados foram organizados e analisados utilizando estatística descritiva por meio do programa Epi Info versão 6.0d, utilizando frequência absoluta e relativa para caracterização dos participantes e das variáveis utilizadas no estudo.

O projeto de pesquisa foi aprovado, parecer $n^{\circ} .416 .831 / 2013$, pelo Comitê de Ética em Pesquisa da Universidade Federal de Sergipe (UFS). Todos os voluntários receberam informações sobre os objetivos e procedimentos do estudo e assinaram o Termo de Consentimento Livre e Esclarecido (TCLE) concordando em participar da pesquisa, de acordo com a Resolução n. 466/2012 do Conselho Nacional de Saúde.

\section{RESULTADOS E DISCUSSÃO}

Dos 120 ACS participantes, 93 (77,5\%) eram do sexo feminino; e 27 (22,5\%), do masculino (tabela 1). Houve equilíbrio no estado civil, com $44,2 \%$ solteiros e $40,0 \%$ casados. Foram encontrados resultados semelhantes, com maior predominância de indivíduos do sexo feminino, casados e com ensino médio completo no estudo de Freitas et al. ${ }^{3}$. Alguns autores relacionaram o trabalho do ACS ao trabalho feminino,

Tabela 1. Perfil socioeconômico dos Agentes Comunitários de Saúde, Lagarto (SE), 2014

\begin{tabular}{|c|c|c|}
\hline Variável & $\mathbf{N}$ & $\%$ \\
\hline \multicolumn{3}{|l|}{ Sexo } \\
\hline Masculino & 27 & 22,5 \\
\hline Feminino & 93 & 77,5 \\
\hline \multicolumn{3}{|l|}{ Estado civil } \\
\hline Solteiro & 53 & 44,2 \\
\hline Casado & 48 & 40,0 \\
\hline Divorciado & 8 & 6,6 \\
\hline Viúvo & 2 & 1,7 \\
\hline Amasiado & 9 & 7,5 \\
\hline \multicolumn{3}{|c|}{ Reside na área de abrangência da unidade } \\
\hline Sim & 100 & 83,3 \\
\hline Não & 20 & 16,7 \\
\hline \multicolumn{3}{|l|}{ Tipo de moradia } \\
\hline Própria, quitada & 87 & 72,5 \\
\hline Própria, ainda pagando & 8 & 6,7 \\
\hline Alugada & 12 & 10,0 \\
\hline Cedida & 12 & 10,0 \\
\hline Outra condição & 1 & 0,8 \\
\hline \multicolumn{3}{|c|}{ Faixa de renda mensal da família } \\
\hline Até 1 salário mínimo & 22 & 18,3 \\
\hline De 1 a 3 & 78 & 65,0 \\
\hline De 3 a 6 & 17 & 14,1 \\
\hline 6 a 9 & 2 & 1,7 \\
\hline > 9 salários & 1 & 0,9 \\
\hline \multicolumn{3}{|l|}{ Escolaridade } \\
\hline Ensino Fundamental & 6 & 5,0 \\
\hline Ensino médio & 82 & 68,3 \\
\hline Técnico & 9 & 7,5 \\
\hline Superior ou graduação & 18 & 15,0 \\
\hline Pós-graduação & 5 & 4,2 \\
\hline
\end{tabular}


considerando o contexto histórico de associação da figura feminina ao cuidado em saúde ${ }^{9,14}$.

Em relação à escolaridade, $68,3 \%$ possuíam ensino médio completo. Embora o trabalho do ACS, a princípio, fosse mais voltado para a educação em saúde, sem a exigência de conhecimento predominantemente técnico e científico, com o decorrer dos anos, houve mudança no perfil desses profissionais com crescente escolarização ${ }^{22}$. Tendo em vista as demandas da comunidade, os ACS do município de Lagarto apontaram a necessidade de busca contínua pelo conhecimento, assim como pela ascensão profissional.

Cada ACS era responsável, em média, por 156 famílias. A maioria $(83,3 \%)$ residia na área de abrangência da Unidade Básica de Saúde (UBS) que atuava; e $72,5 \%$, em imóvel próprio quitado. $\mathrm{O}$ fato do acesso ao cargo de ACS no município ter ocorrido por meio de concurso público, conferindo estabilidade aos trabalhadores, deve contribuir para esse resultado, o que favorece a fixação do profissional e o estabelecimento de vínculo com a comunidade local.

Para o cumprimento das atribuições do ACS, é primordial o acesso ao território e famílias, fazendose necessários meios de locomoção adequados. Nesse sentido, a caminhada acaba sendo o meio mais fácil e econômico para executar as visitas domiciliares. Contudo, longas distâncias e barreiras geográficas podem dificultar o acesso ${ }^{23}$. Pouco mais da metade dos ACS participantes $(50,4 \%)$ relataram utilizar o deslocamento a pé da residência ao trabalho, o que pode gerar insatisfação pela distância das UBS às microáreas, pela ausência de transporte público organizado no município e pelas elevadas temperaturas na maior parte do ano.

Dos $36,7 \%$ que afirmaram realizar algum tipo de atividade física, 45,4\% praticavam em uma frequência de 3 a 4 dias por semana. Os resultados corroboram os achados de Ursine, Trelha e Nunes ${ }^{23}$, nos quais $43,8 \%$ dos ACS relataram algum tipo de prática. Considerando os benefícios da atividade física na prevenção e controle de doenças crônicodegenerativas e melhoria da qualidade de vida, devem-se estimular programas de exercícios físicos voltados aos ACS, que poderiam atuar como multiplicadores em ações de educação em saúde.

Questionados sobre capacitação, 93,3\% relataram participar em, pelo menos, um curso, porém, 46,0\% referiram que raramente estes foram ofertados. Freitas et al. $^{3}$ enfatizaram a importância da qualificação, visando aliar o conhecimento popular, acumulado pela experiência em situações de adoecimento e de assistência precária aos serviços públicos, com o conhecimento técnicocientífico na área da saúde, indispensável para as práticas de promoção da saúde. Ou seja, são necessárias as capacitações frequentes, tendo em vista as demandas da comunidade, bem como a necessidade de conhecimento técnico-científico dos ACS.

Em relação ao domínio salário (compensação) justo e adequado, $47,5 \%$ dos ACS demonstraram insatisfação, com $74,1 \%$ insatisfeitos ou muito insatisfeitos com os salários comparando ao dos colegas, $81,6 \%$ insatisfeitos ou muito insatisfeitos com as recompensas e $57,5 \%$ muito insatisfeitos com os benefícios extras recebidos. Paula et al. ${ }^{17}$ apontaram que tanto os baixos salários como a sobrecarga e desvalorização do trabalho dos ACS geram desmotivação em relação à profissão e que tal situação influencia a satisfação do trabalhador, contribuindo negativamente para o sofrimento e adoecimento, agravado pela recorrente desvalorização da área da saúde pelo poder público, gerando remuneração e benefícios aquém das responsabilidades que os profissionais têm que assumir.

Dentro do domínio condições de trabalho, $27,5 \%$ estavam insatisfeitos com a jornada de trabalho, e $25,0 \%$ estavam satisfeitos; no quesito carga de trabalho, 33,3\% estavam insatisfeitos; e $24,1 \%$, satisfeitos. É válido destacar que a carga de trabalho do ACS é de 40 horas semanais, porém, seu trabalho é direcionado por metas, como o acompanhamento de aproximadamente 150 famílias, possibilitando uma flexibilização da jornada de trabalho, que pode justificar a diversificação nas opiniões a respeito da jornada e carga de trabalho do $\operatorname{ACS}^{24,25}$. 
Tabela 2. Percepções de acordo com o modelo Walton acerca da Qualidade de Vida no Trabalho dos ACS, Lagarto (SE), 2014

\begin{tabular}{|c|c|c|c|c|c|}
\hline Domínio & $\begin{array}{c}\text { Muito } \\
\text { insatisfeito }\end{array}$ & Insatisfeito & $\begin{array}{c}\text { Nem } \\
\text { satisfeitol } \\
\text { Nem } \\
\text { insatisfeito } \\
\end{array}$ & Satisfeito & $\begin{array}{c}\text { Muito } \\
\text { satisfeito }\end{array}$ \\
\hline \multicolumn{6}{|l|}{ Salário justo e adequado } \\
\hline Salário & $28,3 \%$ & $47,5 \%$ & $17,5 \%$ & $5,8 \%$ & $0,8 \%$ \\
\hline Salário comparado ao dos colegas & $25,0 \%$ & $49,1 \%$ & $15,8 \%$ & $9,1 \%$ & $0,8 \%$ \\
\hline Recompensas & $34,1 \%$ & $47,5 \%$ & $6,6 \%$ & $5,0 \%$ & $6,6 \%$ \\
\hline Benefícios extras & $57,5 \%$ & $36,6 \%$ & $1,6 \%$ & $3,3 \%$ & $0,8 \%$ \\
\hline \multicolumn{6}{|l|}{ Condições de trabalho } \\
\hline Jornada de trabalho semanal & $20,0 \%$ & $27,5 \%$ & $21,6 \%$ & $25,0 \%$ & $5,8 \%$ \\
\hline Carga de trabalho & $15,0 \%$ & $33,3 \%$ & $23,3 \%$ & $24,1 \%$ & $4,1 \%$ \\
\hline Uso de tecnologias & $35,8 \%$ & $45,8 \%$ & $12,5 \%$ & $5,8 \%$ & $0 \%$ \\
\hline Salubridade & $25,0 \%$ & $45,8 \%$ & $15,0 \%$ & $12,5 \%$ & $1,6 \%$ \\
\hline Uso de EPIs & $55,0 \%$ & $33,3 \%$ & $8,3 \%$ & $0,8 \%$ & $2,5 \%$ \\
\hline Cansaço causado & $21,6 \%$ & $39,1 \%$ & $20,0 \%$ & $15,0 \%$ & $4,1 \%$ \\
\hline \multicolumn{6}{|l|}{ Uso das capacidades } \\
\hline Autonomia & $12,5 \%$ & $27,5 \%$ & $18,3 \%$ & $32,5 \%$ & $9,1 \%$ \\
\hline Importância da profissão & $3,3 \%$ & $11,6 \%$ & $7,5 \%$ & $50,0 \%$ & $27,5 \%$ \\
\hline Polivalência & $4,1 \%$ & $30,0 \%$ & $23,3 \%$ & $30,8 \%$ & $11,6 \%$ \\
\hline Avaliação de desempenho & $2,5 \%$ & $9,1 \%$ & $16,6 \%$ & $52,5 \%$ & $19,1 \%$ \\
\hline Responsabilidade conferida & $5,0 \%$ & $19,1 \%$ & $12,5 \%$ & $47,5 \%$ & $15,8 \%$ \\
\hline \multicolumn{6}{|l|}{ Oportunidade de crescimento } \\
\hline Crescimento profissional & $29,1 \%$ & $36,6 \%$ & $10,0 \%$ & $17,5 \%$ & $6,6 \%$ \\
\hline Treinamentos & $24,1 \%$ & $40,0 \%$ & $15,0 \%$ & $15,0 \%$ & $5,8 \%$ \\
\hline Situações de demissão & $7,5 \%$ & $32,5 \%$ & $24,1 \%$ & $27,5 \%$ & $8,3 \%$ \\
\hline Incentivo para estudar & $43,3 \%$ & $44,1 \%$ & $4,1 \%$ & $6,6 \%$ & $1,6 \%$ \\
\hline \multicolumn{6}{|l|}{ Integração social } \\
\hline Discriminação & $5,8 \%$ & $22,5 \%$ & $18,3 \%$ & $40,8 \%$ & $12,5 \%$ \\
\hline Relacionamento (colegas e chefes) & $3,3 \%$ & $5,8 \%$ & $8,3 \%$ & $53,3 \%$ & $29,1 \%$ \\
\hline Comprometimento da equipe & $3,3 \%$ & $9,1 \%$ & $16,6 \%$ & $52,5 \%$ & $18,3 \%$ \\
\hline Valorização de ideias e iniciativas & $11,6 \%$ & $30,0 \%$ & $17,5 \%$ & $30,8 \%$ & $10,0 \%$ \\
\hline \multicolumn{6}{|l|}{ Constitucionalismo } \\
\hline Respeito aos direitos & $32,5 \%$ & $43,3 \%$ & $11,6 \%$ & $10,0 \%$ & $2,5 \%$ \\
\hline Liberdade de expressão & $11,6 \%$ & $25,0 \%$ & $18,3 \%$ & $35,0 \%$ & $10,0 \%$ \\
\hline Normas e regras & $9,1 \%$ & $36,6 \%$ & $25,8 \%$ & $20,8 \%$ & $7,5 \%$ \\
\hline Respeito a individualidade & $3,3 \%$ & $10,8 \%$ & $19,1 \%$ & $50,0 \%$ & $16,6 \%$ \\
\hline \multicolumn{6}{|l|}{ Trabalho e vida } \\
\hline Trabalho e vida & $3,3 \%$ & $15,8 \%$ & $11,6 \%$ & $50,8 \%$ & $18,3 \%$ \\
\hline Trabalho e lazer & $7,5 \%$ & $18,3 \%$ & $15,8 \%$ & $45,8 \%$ & $12,5 \%$ \\
\hline Trabalho e descanso & $5,0 \%$ & $27,5 \%$ & $19,1 \%$ & $38,3 \%$ & $10,0 \%$ \\
\hline \multicolumn{6}{|l|}{ Relevância social } \\
\hline Orgulho & $4,1 \%$ & $7,5 \%$ & $5,0 \%$ & $50,0 \%$ & $33,3 \%$ \\
\hline Imagem da secretaria & $27,5 \%$ & $37,5 \%$ & $19,1 \%$ & $13,3 \%$ & $2,5 \%$ \\
\hline Integração comunitária & $23,3 \%$ & $31,6 \%$ & $29,1 \%$ & $11,6 \%$ & $4,1 \%$ \\
\hline Qualidade dos serviços prestados & $20,0 \%$ & $40,0 \%$ & $28,3 \%$ & $9,1 \%$ & $2,5 \%$ \\
\hline Política de recursos humanos & $26,8 \%$ & $40,3 \%$ & $17,6 \%$ & $12,6 \%$ & $2,5 \%$ \\
\hline
\end{tabular}


Fatores de sobrecarga no trabalho prejudicam a qualidade com que as atividades são realizadas e, consequentemente, a QVT. Carga horária de trabalho, metas a serem cumpridas, falta de privacidade e liberdade, local de moradia, interação com os demais profissionais e com moradores da comunidade são alguns desses fatores apontados como barreiras no trabalho do ACS ${ }^{17,26}$.

Ainda no domínio condições de trabalho, os participantes estavam muito insatisfeitos ou insatisfeitos em relação ao uso de tecnologias $(81,6 \%)$, assim como com as condições salubres do trabalho $(70,8 \%)$; e $88,3 \%$, com os equipamentos de segurança e proteção individuais e coletivos disponibilizados.

No estudo de Nascimento e David ${ }^{27}$, sobre os riscos no trabalho do ACS, destacaram-se os físicos, como a exposição aos raios solares. Os profissionais estão propensos a desenvolver câncer de pele, em função de ter a visita domiciliar como atividade laboral essencial, associado ao clima quente e seco no Brasil. Por outro lado, também ocorre a exposição à umidade, nos períodos de chuvas e em locais úmidos.

Em relação ao domínio relevância social e importância do trabalho, nos quesitos qualidade dos serviços prestados, $60 \%$ estavam insatisfeitos ou muito insatisfeitos. Pouco mais de $66,8 \%$ se sentiam da mesma forma em relação ao tratamento recebido pela secretaria. O setor saúde, como prestador de serviços, deveria lançar mão de recursos para viabilizar um melhor desempenho, com uma melhor satisfação, tanto dos usuários quanto dos servidores.

Por outro lado, no domínio integração social, nos quesitos relacionamento com os colegas $\mathrm{e}$ chefes no trabalho e comprometimento da equipe, a maioria estava satisfeita. $O$ estudo de Medeiros et al. $^{28}$ estabeleceu uma análise qualitativa, identificando a existência de ajuda mútua entre os agentes, bom relacionamento e cumplicidade entre eles e a equipe da ESF. Dessa forma, o trabalho torna-se significativo pela existência de relações de parceria entre os membros da equipe.

No domínio oportunidade de crescimento, os ACS estavam muito insatisfeitos ou insatisfeitos com a possibilidade de crescimento profissional $(65,7 \%)$, bem como com os treinamentos realizados $(64,1 \%)$. Além de estarem insatisfeitos ou muito insatisfeitos na categoria respeito aos direitos do trabalhador $(75,8 \%)$.

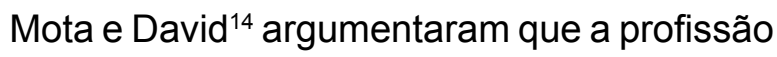
de ACS possibilita o acesso a um conhecimento, um saber novo, por meio de capacitações para o trabalho. A atuação em equipe multiprofissional e a formação em serviço conferem aos ACS uma sensação de pertencimento a uma equipe de saúde e uma sensação de prestígio social, por poder diferenciar seu conhecimento do conhecimento popular.

Por outro lado, a indefinição é marcante no seu cotidiano de trabalho, não somente porque não Ihe seja dado, a priori, um corpo de conhecimentos bem definidos, e, sim, pelo fato de que o seu trabalho se constitui na pluralidade, em encontros e desencontros, nos quais ele afeta e é afetado. O trabalho do ACS é, em si, potência, diversidade, indefinição, busca, rupturas, contratualidades e fugas $^{29}$.

O grau de insatisfação dos ACS com as capacitações e treinamentos realizados, considerados insuficientes, pode estar associado a dificuldades enfrentadas em situações na sua rotina que exigem conhecimentos técnico-científicos, como dúvidas de usuários, como discutido em Garcia et al. ${ }^{12}$. Além disso, sua insatisfação com salário, fornecimento de equipamentos e proteção e segurança, condições no trabalho, possibilidade de crescimento profissional podem estar relacionados 
com a insatisfação no domínio respeito aos direitos do trabalhador.

Por outro lado, mais de $70 \%$ dos participantes sentiam-se satisfeitos ou muito satisfeitos com a importância da profissão, com as avaliações de desempenho realizadas e com a responsabilidade conferida. Isso sugere que, apesar das dificuldades apontadas no estudo, o profissional sente-se realizado em ser ACS, considerando a possibilidade de ajudar a comunidade em que reside e contribuir para a melhoria da saúde da comunidade ${ }^{26}$. Barroso e Guerra ${ }^{30}$ também identificaram que mais da metade dos ACS apresentavam realização pessoal satisfatória $(58,3 \%)$. Ponderaram, ainda, que um dos aspectos que contribuiu para a manutenção da percepção de realização pessoal veio do tipo de trabalho realizado, que permitiu ao ACS sentir-se útil para a comunidade em que vivia e atuava.

\section{CONCLUSÃO}

O trabalho do ACS é caracterizado pela intermediação do acesso a direitos sociais para a comunidade em que vive e atua, podendo levar à sobrecarga física e mental. Os resultados do estudo demonstraram que a maioria dos participantes estava insatisfeita ou muito insatisfeita com o salário, benefícios extras, distribuição dos EPI, oportunidade de crescimento profissional, treinamentos e respeito aos direitos do trabalhador. Por outro lado, estavam satisfeitos ou muito satisfeitos com a importância do trabalho, relacionamento com colegas e chefes, comprometimento com o trabalho, respeito à individualidade e relevância social.

Com base nos achados, sugerem-se ações destinadas à melhoria das condições de vida e trabalho desses trabalhadores, com a garantia de condições salubres no trabalho e o fornecimento de EPI, além de um olhar atencioso do poder público local para esses indivíduos/ profissionais e para relevância do seu trabalho com a população do município, possibilitando ações contínuas de qualificação, que contribuam para o crescimento pessoal e profissional e forneçam os conhecimentos necessários para execução das atividades diárias. Por outro lado, nesse processo, faz-se necessário um movimento de busca de autonomia, de fortalecimento e valorização enquanto cidadãos e categoria profissional que potencialize o papel do ACS na sociedade. 


\section{REFERÊNCIAS}

1. Carneiro CCG, Martins MIC. Novos modelos de gestão do trabalho no setor público de saúde e o trabalho do agente comunitário de saúde. Trab. educ. saúde. 2015 abr;13(1):45-66.

2. Moura FFS, Silva CRC. Afetividade e seus sentidos no trabalho do agente comunitário de saúde. Physis. 2015 set;25(3):993-1010.

3. Freitas LM, Coriolano-Marinus MWL, Lima LS, Ruiz-Moreno L. Formação dos agentes comunitários de saúde no município de Altamira (PA), Brasil. ABCS Health Sci. 2015;40(3):171-177.

4. Fonseca AF, Machado FRS, Bornstein VJ, Pinheiro R. Avaliação em saúde e repercussões no trabalho do agente comunitário de saúde. Texto contexto - enferm. 2012 jul./set; 21(3):519-527.

5. Cardoso AS, Nascimento MC. Comunicação no Programa Saúde da Família: o agente de saúde como elo integrador entre a equipe e a comunidade. Ciênc. saúde coletiva. 2010 jun;15(1):1509-1520.

6. Santos LPG, Fracolli LA. O Agente Comunitário de Saúde: possibilidades e limites para a promoção da saúde. Rev Esc Enferm USP. 2010 mar;44(1):76-83.

7. Vidal SV, Motta LCS, Siqueira-Batista R. Agentes comunitários de saúde: aspectos bioéticos e legais do trabalho vivo. Saúde Soc. 2015 mar;24(1):129-140.

8. Silva CRC, Chiaperini PL, Frutuoso MFP, Morell MGGP. Extensão universitária e prática dos agentes comunitários de saúde: acolhimento e aprendizado cidadão. Saúde soc. 2014 jun;23(2):677-688.

9. Santos ÍER, Vargas MM, Reis FP. Estressores laborais em agentes comunitários de saúde. rPOT. 2014 set;14(3):324-335.

10. Brasil. Lei n. 11.350, de 05 de outubro de 2006. Diário Oficial da União, Brasília, DF, 06 outubro 2006. Sec.1, p.1.

11. Resende MC, Azevedo EGS, Lourenço LR, Faria LS, Alves NF, Farina NP et al. Saúde mental e ansiedade em agentes comunitários que atuam em saúde da família em Uberlândia (MG, Brasil). Ciênc. saúde coletiva. 2011 abr;16(4):2115-2122.

12. Garcia ACP, Lima RCD, Galavote HS, Coelho APS, Vieira ECL, Silva RC, et al. Agente comunitário de saúde no Espírito Santo: do perfil às atividades desenvolvidas. Trab. educ. saúde. 2017 jan./abr;15(1):283300.

13. Gomes KO, Cotta RMM, Mitre SM, Batista RS, Cherchiglia ML. O agente comunitário de saúde e a consolidação do Sistema Único de Saúde: reflexões contemporâneas. Physis. 2010 Dez;20(4):1143-1164.

14. Mota RRA, David HMSL. A crescente escolarização do agente comunitário de saúde: uma indução do processo de trabalho? Trab. educ. saúde. 2010 out;8(2):229-248. 
15. Menegussi JM, Ogata MN, Rosalini MHP. O agente comunitário de saúde como morador, trabalhador e usuário em São Carlos, São Paulo. Trab. educ. saúde. 2014 abr.;12(1):87-106.

16. Mascarenhas $\mathrm{CHM}$, Prado FO, Fernandes $\mathrm{MH}$. Fatores associados à qualidade de vida de Agentes Comunitários de Saúde. Ciênc. saúde coletiva. 2013 mai.;18(5):1375-1386.

17. Paula ÍR, Marcacine PR, Castro SS, Walsh IAP. Work ability, musculoskeletal symptoms and quality of life among community health workers in Uberaba, Minas Gerais, Brazil. Saude soc. 2015 mar;24(1):152-164. 18. Portugal FB, Campos MR, Gonçalves DA, Mari JJ, Fortes SLCLima. Qualidade de vida em pacientes da atenção primária do Rio de Janeiro e São Paulo, Brasil: associações com eventos de vida produtores de estresse e saúde mental. Ciênc. saúde coletiva. 2016 Fev;21(2):497-508.

19. Rueda FJM, Lima RC, Raad AJ. Qualidade de vida e satisfação no trabalho: relação entre escalas que avaliam os construtos. Boletim de psicologia. 2014 dez.;64(141):129-141.

20. Padilha V. Qualidade de vida no trabalho num cenário de precarização: a panaceia delirante. Trab. educ. saúde. 2009 nov;7(3):549-563.

21. Marques AL, Borges R, Reis IC. Mudança organizacional e satisfação no trabalho: um estudo com servidores públicos do estado de Minas Gerais. Revista de Administração Pública. 2016 fev;50(1):41-58.

22. Musse JO, Marques RS, Lopes FRL, Monteiro KS, Santos SC. Avaliação de competências de Agentes Comunitários de Saúde para coleta de dados epidemiológicos. Ciênc. saúde coletiva. 2015 fev;20(2):525536.

23. Ursine BL, Trelha CS, Nunes EFPA. O Agente Comunitário de Saúde na Estratégia de Saúde da Família: uma investigação das condições de trabalho e da qualidade de vida. Rev Bras Saude Ocup. 2010 dez;35(122):327-339.

24. Brasil. Decreto 8.474 de 22 de junho de 2015. Diário Oficial da União: Brasília, DF, 23 junho 2015. N.117, p.10.

25. Ministério da Saúde. Guia Prático Saúde da Família. Brasília: Ministério da Saúde; 2001. 128p.

26. Costa EM, Ferreira DLA. Percepções e motivações de agentes comunitários de saúde sobre o processo de trabalho em Teresina, Piauí. Trab. educ. saúde. 2011 nov;9(3):461-478.

27. Nascimento GM, David, HMSL. Avaliação de riscos no trabalho dos Agentes Comunitários de Saúde: um processo participativo. Revista enfermagem, UERJ. 2008 out./dez;16(4):550-556.

28. Medeiros RLR, Andrade AMBA, Fernandes AFC, Almeida NMGS. O Agente Comunitário de Saúde como agente de mudança sociocultural. Rede Observatório de Recursos Humanos em Saúde. 2006.

29. Galavote HS, Franco TB, Lima RCD, Belizário AM. Alegrias e tristezas no cotidiano de trabalho do agente comunitário de saúde: cenários de paixões e afetamentos. Interface - Comunicação, Saúde, Educação. 2013 ago;17(46): 575-586.

30. Barroso SM, Guerra ARP. Burnout e qualidade de vida de agentes comunitários de saúde de Caetanópolis (MG). Cad. Saude Colet. 2013 set;21(3):338-345. 